\title{
Microlens MASSES From 1-D PaRAllaXes AND Heliocentric Proper Motions
}

\author{
ANDREW Gould \\ Department of Astronomy, Ohio State University, 140 W. 18th Ave., Columbus, OH 43210, USA \\ gould@astronomy. ohio-state.edu
}

Received August 5, 2014; accepted October 4, 2014

\begin{abstract}
One-dimensional (1-D) microlens parallaxes can be combined with heliocentric lens-source relative proper motion measurements to derive the lens mass and distance, as suggested by Ghosh et al. (2004). Here I present the first mathematical anlysis of this procedure, which I show can be represented as a quadratic equation. Hence, it is formally subject to a two-fold degeneracy. I show that this degeneracy can be broken in many cases using the relatively crude 2-D parallax information that is often available for microlensing events. I also develop an explicit formula for the region of parameter space where it is more difficult to break this degeneracy. Although no mass/distance measurements have yet been made using this technique, it is likely to become quite common over the next decade.
\end{abstract}

Key words: astrometry — gravitational microlensing — planets — stars: fundamental parameters (masses)

\section{INTRODUCTION}

Even at the dawn of microlensing experiments 20 years ago, it was already realized that vastly more microlensing events will yield one-dimensional (1-D) microlens parallaxes than full (2-D) parallaxes (Gould et al. 1994). However, these 1-D parallaxes remain largely unexploited.

The amplitude of the microlens parallax vector $\boldsymbol{\pi}_{\mathrm{E}}$ is the ratio of the lens-source relative parallax $\pi_{\text {rel }}$ to the angular Einstein radius $\theta_{\mathrm{E}}$, while its direction is set by the lens-source relative proper motion $\boldsymbol{\mu}$,

$$
\boldsymbol{\pi}_{\mathrm{E}}=\frac{\pi_{\mathrm{rel}}}{\theta_{\mathrm{E}}} \frac{\boldsymbol{\mu}}{\mu} .
$$

The numerator of the first ratio quantifies the apparent angular lens-source displacement as the observer changes position, while the denominator translates this angular scale into the scale of microlensing phenomena. The second term appears because the evolution of microlensing effects due to parallax depends on the direction of lens-source relative motion. See Figure 1 of Gould \& Horne (2013).

Note that from the definition of $\theta_{\mathrm{E}}$,

$$
\theta_{\mathrm{E}}^{2} \equiv \kappa M \pi_{\text {rel }}, \quad \kappa \equiv \frac{4 G}{c^{2} \mathrm{AU}} \simeq 8.1 \frac{\mathrm{mas}}{M_{\odot}},
$$

measurement of both $\theta_{\mathrm{E}}$ and $\pi_{\mathrm{E}}$ directly leads to determinations of the lens mass and relative parallax

$$
M=\frac{\theta_{\mathrm{E}}}{\kappa \pi_{\mathrm{E}}}, \quad \pi_{\mathrm{rel}}=\theta_{\mathrm{E}} \pi_{\mathrm{E}}
$$

The reason that 1-D parallaxes are easier to measure is that, to the extent that $\boldsymbol{\mu}$ is parallel to the direction of Earth's acceleration (projected on the sky at

Corresponding Author: A. Gould the peak of the event) $\hat{\mathbf{n}}_{a}$, it causes the event evolution to speed up or slow down as it progresses, thereby inducing an asymmetric distortion on the lightcurve. Since microlensing lightcurves are otherwise intrinsically symmetric, such distortions are easily measured. By contrast, to the extent that $\boldsymbol{\mu}$ is perpendicular to $\hat{\mathbf{n}}_{a}$, it gives rise to a symmetric distortion, which is much more difficult to disentangle from other symmetric parameters. Hence, we define

$$
\pi_{\mathrm{E}, \|} \equiv \boldsymbol{\pi}_{\mathrm{E}, \mathrm{geo}} \cdot \hat{\mathbf{n}}_{a}
$$

and $\pi_{\mathrm{E}, \perp}=\left(\pi_{\mathrm{E}}^{2}-\pi_{\mathrm{E}, \|}^{2}\right)^{1 / 2}$ (see Gould 2004 for sign conventions).

A very important notational point for the current work is that $\boldsymbol{\pi}_{\mathrm{E}}$ is subscripted with "geo" (to indicate geocentric frame at the peak of the event) whereas $\pi_{\mathrm{E}}$ is not. This is because the magnitude of this vector does not depend on the frame of reference, but the direction does (due to explicit dependence on $\boldsymbol{\mu} / \mu$ in Equation (1)).

Despite their predicted ubiquity, 1-D parallaxes are almost never reported in the literature. Among the handful of exceptions (Park et al. 2004; Ghosh et al. 2004; Jiang et al. 2004; Dong et al. 2009; Muraki et al. 2011), the first three reports are due to the novelty of the phenomenon and the last two are due to the fact that the actual value of $\pi_{\mathrm{E}, \|}$ was so large that $\pi_{\mathrm{E}}$ (and so $M$ ) could be reasonably estimated despite the poor measurement of $\pi_{\mathrm{E}, \perp}$.

Nevertheless, as first pointed out by Ghosh et al. (2004), 1-D microlens parallaxes could yield microlens mass (and distance) measurements simply by making a late-time measurement of the heliocentric proper motion. Stated in the most naive way, knowing one component $\left(\pi_{\mathrm{E}, \|}\right)$ of the vector $\boldsymbol{\pi}_{\mathrm{E}}$ (from the microlensing 
event) and its direction $\boldsymbol{\mu} / \mu$ (from late-time astrometry), one can extract the amplitude of the full vector $\pi_{\mathrm{E}}$. Then knowing the Einstein timescale $t_{\mathrm{E}}$ (again from the event) and the amplitude of proper motion $\mu$, one can determine the angular Einstein radius $\theta_{\mathrm{E}}=\mu t_{\mathrm{E}}$.

The problem with this naive reasoning, as already recognized by Ghosh et al. (2004), is that $\pi_{\mathrm{E}, \|}$ and $t_{\mathrm{E}}$ are derived in the geocentric frame, whereas $\boldsymbol{\mu}$ is measured in the heliocentric frame. The relation between these frames, as noted by Ghosh et al. (2004) but in a form closer to that preferred by Janczak et al. (2010), is

$$
\boldsymbol{\mu}_{\mathrm{hel}}=\boldsymbol{\mu}_{\mathrm{geo}}+\boldsymbol{\mu}_{\oplus} \pi_{\mathrm{rel}}=\frac{\theta_{\mathrm{E}}}{t_{\mathrm{E}}} \frac{\boldsymbol{\pi}_{\mathrm{E}, \mathrm{geo}}}{\pi_{\mathrm{E}}}+\boldsymbol{\mu}_{\oplus} \pi_{\mathrm{E}} \theta_{\mathrm{E}},
$$

where $\boldsymbol{\mu}_{\oplus} \equiv \mathbf{v}_{\oplus, \perp} / \mathrm{AU}$ and $\mathbf{v}_{\oplus, \perp}$ is the transverse velocity of Earth in the frame of the Sun at the peak of the event, projected on the plane of the sky.

However, in the intervening 10 years, there has been essentially no work aimed at understanding Equation (5). For example, it has not even been recognized that this equation leads to a quadratic equation in $\pi_{\mathrm{E}, \perp}$, which therefore has two (or zero) real solutions.

Nevertheless, many changes in the observational landscape are leading to radical improvements for the prospects of implementing the original suggestion of Ghosh et al. (2004). First is the simple fact that the massive search for events made possible by the inauguration of the Optical Gravitational Lensing Experiment (OGLE-III) survey in 2002 are now more than 10 years old, implying that sources and lenses have already substantially separated. Second, the Giant Magellan Telescope (GMT) is already under construction and likely to be operational within another 10 years. When it is, its FWHM in $J$ band will be about 11 mas, meaning that sources and lenses separated by just 2 FWHM $(22$ mas) will be eligible for very good $\boldsymbol{\mu}_{\text {hel }}$ measurements. Third, microlensing event detections have already increased dramatically since 2011 with the inauguration of the OGLE-IV survey and are likely to accelerate further in 2015 with the inauguration of the new Korea Microlensing Telescope Network (KMTNet) of $1.6 \mathrm{~m}$ telescopes with $4 \mathrm{deg}^{2}$ cameras on three continents. Since typical lens-source proper motions are $\mu \sim 4$ mas yr $^{-1}$, many of these events will be accessible to GMT. Finally, it is quite plausible that new space-based widefield survey telescopes, like Euclid and WFIRST will survey essentially the entire bulge microlensing field at roughly the same epoch as GMT first light. Since these have much smaller apertures than GMT, their FWHM will be much larger (roughly 140 mas for Euclid and 110 for WFIRST). However, their greater point-spreadfunction stability will permit proper-motion measurements at 1 FWHM (rather than 2), and their wider (i.e., systematic) coverage will permit a systematic search for relatively high proper-motion archival events over a very large field.

Of course, once such data are obtained, the analysis will proceed by simultaneous fitting of the microlensing and astrometric data. However, a proper analytic investigation of Equation (5) is still very important for understanding what can be learned from such observations, which is the central motivation for taking them.

\section{QUADRATIC FORM}

Simply taking the ratio of the two components (perpendicular and parallel to $\hat{\mathbf{n}}_{a}$ ) of $\boldsymbol{\mu}_{\text {hel }}$ (Equation (5)) yields the tangent of the angle $\phi$ of this vector relative to $\hat{\mathbf{n}}_{a}$,

$$
\tan \phi \equiv \frac{\mu_{\mathrm{hel}, \perp}}{\mu_{\mathrm{hel}, \|}}=\frac{\pi_{\mathrm{E}, \perp}+\theta_{\oplus, \perp} \pi_{\mathrm{E}}^{2}}{\pi_{\mathrm{E}, \|}+\theta_{\oplus, \|} \pi_{\mathrm{E}}^{2}} ; \quad \boldsymbol{\theta}_{\oplus} \equiv \boldsymbol{\mu}_{\oplus} t_{\mathrm{E}}
$$

Note that $\theta_{\oplus}=0.23\left(v_{\oplus, \perp} / 20 \mathrm{~km} \mathrm{~s}^{-1}\right)\left(t_{\mathrm{E}} / 20\right.$ day $)$. Then writing $\pi_{\mathrm{E}}^{2}=\pi_{\mathrm{E}, \|}^{2}+\pi_{\mathrm{E}, \perp}^{2}$ and rearranging terms yields

$$
A \pi_{\mathrm{E}, \perp}^{2}-\pi_{\mathrm{E}, \perp}+C=0
$$

where

$$
A \equiv \theta_{\oplus, \|} \tan \phi-\theta_{\oplus, \perp} ; \quad C \equiv A \pi_{\mathrm{E}, \|}^{2}+\pi_{\mathrm{E}, \|} \tan \phi
$$

which has the solutions

$$
\pi_{\mathrm{E}, \perp}=\frac{1 \pm \sqrt{1-4 A C}}{2 A} .
$$

\subsection{Limit of $4 A C \ll 1$}

In the limit $4 A C \ll 1$, the two solutions of Equation (9) can be approximated as

$$
\pi_{\mathrm{E}, \perp}=C(1+A C+\ldots) \quad(4 A C \ll 1)
$$

and

$$
\pi_{\mathrm{E}, \perp}=\frac{1}{A}-C+\ldots \quad(4 A C \ll 1, \quad \text { alternate }) .
$$

Note that both solutions are perfectly valid. The leading term in the first solution corresponds to ignoring the first term in Equation (7) and yields "small" $\pi_{\mathrm{E}, \perp}$, while the leading term in the second solution corresponds to ignoring the last term in Equation (7) and yields "large" $\pi_{\mathrm{E}, \perp}$.

In practice, there will be some information about $\pi_{\mathrm{E}, \perp}$ from the microlensing event, which may well be adequate to break this degeneracy. The important point is, however, that these two solutions are likely to correspond to distinct minima, so that they will not both automatically be probed by simple downhill minimization: they must both be explicitly checked. This situation is similar to the "jerk-parallax" degenerate solutions, which also constitute the roots of quadratic equation in $\pi_{\mathrm{E}, \perp}$. See Equation (20) of Gould (2004). In fact, prior to the discovery of this degeneracy, Alcock et al. (2001) found one of the two solutions by downhill minimization. Gould (2004) then found the second solution from the symmetries of the quadratic equation, which turned out to have equally good $\chi^{2}$. Subsequent astrometric measurements by Drake et al. (2004) showed that the second solution was correct (Gould et al. 2004). 


\subsection{General Case}

Equation (9) can be reformulated to eliminate " $C$ " in favor of direct observables

$$
\pi_{\mathrm{E}, \perp}=\frac{1 \pm \sqrt{\sec ^{2} \phi-\left(2 A \pi_{\mathrm{E}, \|}+\tan \phi\right)^{2}}}{2 A} .
$$

Thus, if $2 A \pi_{\mathrm{E}, \|} \sim \sec \phi-\tan \phi$, then the argument under the radical (discriminant) is close to zero and there is a danger that the error ellipses from the two solutions merge and/or cannot be distinguished by the microlensing data. Thus checking for this approximate equality is an important diagnostic.

If the source lies on the ecliptic, then $\theta_{\oplus, \perp}=0$. Because microlensing fields lie close to the ecliptic, this can often be a useful approximation. In this case

$$
\begin{aligned}
& \pi_{\mathrm{E}, \perp}= \frac{\cot \phi \pm \sqrt{\csc ^{2} \phi-\left(1+2 \theta_{\oplus, \|} \pi_{\mathrm{E}, \|}\right)^{2}}}{2 \theta_{\oplus, \|}} ; \\
&\left(\theta_{\oplus, \perp} \equiv 0\right) .
\end{aligned}
$$

In this form, it is clear that as $\phi$ tends toward $\pm \pi / 2$, the discriminant will very likely be small (and hence the solution prone to degeneracy).

Another key point is that even if $\pi_{\mathrm{E}, \|}=0$, it may still be possible to measure $\pi_{\mathrm{E}, \perp}$ (and so $\pi_{\mathrm{E}}$ ) from the direction of $\boldsymbol{\mu}_{\text {hel }}$. This would not be possible from the naive perspective outlined in Section 1. Explicitly,

$$
\pi_{\mathrm{E}, \perp}=\frac{1}{A}=\frac{1}{\theta_{\oplus, \|} \tan \phi-\theta_{\oplus, \perp}} \quad\left(\pi_{\mathrm{E}, \|}=0\right) .
$$

This is important, particularly if there are widefield high-resolution survey data (from e.g., Euclid or WFIRST) for which special observations of "lowprobability" (i.e., $\pi_{\mathrm{E}, \|} \sim 0$ ) targets are not required. However, events that have high priority (such as those with planetary events) with $\pi_{\mathrm{E}, \|} \sim 0$ could be targeted for individual observations to measure $\boldsymbol{\mu}_{\text {hel }}$, particularly if the planetary-event lightcurve indicated a high geocentric scalar proper motion $\mu_{\text {geo }}$ (Henderson et al. 2014).

\section{Error ANALYSis}

While $\mathbf{v}_{\oplus, \perp}$ is known exactly, none of the quantities entering Equation (7) are known exactly. In particular, $\boldsymbol{\theta}_{\oplus}=\mathbf{v}_{\oplus, \perp} t_{\mathrm{E}} / \mathrm{AU}$, and $t_{\mathrm{E}}$ is a measured quantity from the event. Similarly, $\pi_{\mathrm{E}, \|}$ is measured from the event, while $\tan \phi$ comes from the proper motion measurement. However, in most cases, $\pi_{\mathrm{E}, \|}$ will be measured with substantially worse precision than the other quantities. Hence, it is useful to approximate the others as "known perfectly" and ask how the uncertainty in $\pi_{\mathrm{E}, \perp}$ depends on the measurement of $\pi_{\mathrm{E}, \|}$. To determine this, I differentiate Equation (7) and find

$$
\frac{\delta \pi_{\mathrm{E}, \perp}}{\delta \pi_{\mathrm{E}, \|}}=\frac{\tan \phi+2 A \pi_{\mathrm{E}, \|}}{1-2 A \pi_{\mathrm{E}, \perp}} .
$$

Clearly the appearance of $\pi_{\mathrm{E}, \perp}$ in the denominator of the right hand side reflects a fundamental shortcoming of making a linearized analysis of an intrinsically nonlinear problem. Nevertheless, this expression points to the possibility of a strong degeneracy if $\pi_{\mathrm{E}, \perp} \sim 1 / 2 \mathrm{~A}$. Note from Equation (9) that this corresponds to the discriminant $(1-4 A C)$ being close to zero ${ }^{\mathbf{1}}$. Recall from Section 2.2 that this is the same region of solution space that is potentially most sensitive to the discrete degeneracy. Therefore, measurements of $\pi_{\mathrm{E}, \|} \sim 1 / 2 \mathrm{~A}$ are particularly problematic. Note, however, from comparison with Equation (14) that this degeneracy specifically does not apply to the $\pi_{\mathrm{E}, \perp}=0$ case.

\section{INFORMATION FROM DIRECT IMAGING}

Of course, if the lens is separately imaged from the source, it is possible in principle to make a photometric estimate of its mass and distance. However, such measurements face a number of challenges. First, the photometry will most often be done in the near-infrared, a spectral region for which reddening is highly degenerate with intrinsic temperature. Second, even if the lens is in the Galactic bulge (and so behind essentially all the dust, whose extinction properties can then be measured from nearby clump stars, e.g., Nataf et al. 2013), even main-sequence stars can differ in mass by several tens of percent at fixed color. Finally, and most fundamentally, most stars are in binaries, and for a significant minority of cases it will be the lower-mass component that gives rise to the microlensing event (because event rate scales as $M^{1 / 2}$ ), while the more massive component will be seen by direct imaging (because light is a high power of mass).

Note that while in high-resolution imaging of microlensing lens/source pairs carried out to date it has often been possible to rule out binary companions over most of parameter space, this will not be possible in the imaging of more generic events in the future. For example, Batista et al. (2014) were able to rule out companions closer than 11 mas. This corresponded to physical separations less than 80 AU. However, the basis for this limit was that the event (MOA-2011-BLG-293) had very high magnification, making it very sensitive to binary companions. By contrast, typical events with 1-D parallaxes will not be high-magnification, implying that microlensing-based constraints on companions will be very weak.

Thus, overall, mass/distance determinations from $\left(\pi_{\mathrm{E}, \|}, \boldsymbol{\mu}_{\text {hel }}\right)$ will be more accurate than those derived from photometry, and in a substantial minority of cases there will be significant disagreement due to lensing by secondary components of binaries. It is true that in these cases the initial $\boldsymbol{\mu}_{\text {hel }}$ measurement will be in error due to an implicit assumption that the primary and the source were initially aligned, whereas actually the alignment was with the unseen (or barely seen) secondary. However, once these cases are identified, they can be rectified by a second epoch of imaging, which

\footnotetext{
${ }^{\mathbf{1}}$ It is a generic property of quadratic equations that if the coefficients depend on some quantity $q$, then the ratio of the error in the solution to the error in $q$ diverges as the discriminant approaches zero
} 
would directly measure the proper motion of the primary. This will differ from the proper motion of the secondary (i.e., the lens) due to orbital motion, but usually by an amount that is very small compared to the lens-source proper motion itself.

In brief, photometric mass/distance estimates can be an important check on estimates derived from a combination of 1-D parallaxes and heliocentric proper motions, but they will generally be less precise and less accurate.

\section{Conclusion}

Determinations of microlens masses from the combination of 1-D microlens parallax $\left(\pi_{\mathrm{E}, \|}\right)$ and heliocentric proper motion $\left(\boldsymbol{\mu}_{\text {hel }}\right)$ are likely to become quite common over the next decade. I have shown that solutions derived from such data are in the form of a quadratic equation and therefore have an intrinsic two-fold degeneracy. This degeneracy may be broken by microlensing data, which weakly constrain $\pi_{\mathrm{E}, \perp}$ even when they are unable to measure it precisely. The degeneracy is most severe when the discriminant of the quadratic equation is near zero. In this case, each solution separately has large errors, so that the two solutions may merge together. Photometric mass/distance estimates can be an important check on mass/distance determinations based on 1-D parallaxes and heliocentric proper motions, but are overall less accurate and less precise.

\section{ACKNOWLEDGMENTS}

I thank Radek Poleski for stimulating discussions. This work was supported by NSF grant AST 1103471 and NASA grant NNX12AB99G.

\section{REFERENCES}

Alcock, C., Allsman, R. A., Alves, D. R., et al. 2001, Direct Detection of a Microlens in the Milky Way, Nature, 414, 617
Batista, V., Beaulieu, J.-P., Gould, A., Bennett, D. P., Yee, J. C., Fukui, A., Sumi, T., \& Udalski, A. 2014, MOA2011-BLG-293Lb: First Microlensing Planet Possibly in the Habitable Zone, ApJ, 780, 54

Dong, S., Gould, A., Udalski, A., et al. 2009, OGLE-2005BLG-071Lb, the Most Massive M Dwarf Planetary Companion?, ApJ, 695, 970

Drake, A. J., Cook, K. H., \& Keller, S. C. 2004, Resolving the Nature of the Large Magellanic Cloud Microlensing Event MACHO-LMC-5, ApJ, 607, L29

Ghosh, H., DePoy, D. L., Gal-Yam, A., et al. 2004, Potential Direct Single-Star Mass Measurement, ApJ, 615, 450

Gould, A. 2000, A Natural Formalism for Microlensing, ApJ, 542, 785

Gould, A. 2004, Resolution of the MACHO-LMC-5 Puzzle: The Jerk-Parallax Microlens Degeneracy, ApJ, 606, 319

Gould, A., Bennett, D. P., \& Alves, D. R. 2004, The Mass of the MACHO-LMC-5 Lens Star, ApJ, 614, 404

Gould, A., \& Horne, K. 2013, Kepler-Like Multi-Plexing for Mass Production of Microlens Parallaxes, ApJL, 779, L28

Gould, A., Miralda-Escudé, J., \& Bahcall, J. N. 1994, Microlensing Events: Thin Disk, Thick Disk, or Halo?, ApJ, 423, L105

Henderson, C. B., Park, H., Sumi, T., et al. 2014, Candidate Gravitational Microlensing Events for Future Direct Lens Imaging, ApJ, 794, 71

Janczak, J., Fukui, A., Dong, S., et al. 2010, Sub-Saturn Planet MOA-2008-BLG-310Lb: Likely to be in the Galactic Bulge, ApJ, 711, 731

Jiang, G., DePoy, D. L., Gal-Yam, A., et al. 2004, OGLE2003-BLG-238: Microlensing Mass Estimate of an Isolated Star, ApJ, 617, 1307

Muraki, Y., Han, C., Bennett, D. P., et al. 2011, Discovery and Mass Measurements of a Cold, 10 Earth Mass Planet and Its Host Star, ApJ, 741, 22

Nataf, D. M., Gould, A., Fouqué, P., et al. 2013, Reddening and Extinction toward the Galactic Bulge from OGLEIII: The Inner Milky Way's RV 2.5 Extinction Curve, ApJ, 769, 88

Park B.-G., DePoy, D. L., Gaudi, B. S., et al. 2004, MOA 2003-BLG-37: A Bulge Jerk-Parallax Microlens Degeneracy, ApJ, 609, 166 